\title{
The role of the nasal allergen provocation test in local allergic rhinitis cases: a preliminary report
}

\author{
Edyta Krzych-Fatta' ${ }^{1}$ Konrad Furmańczyk ${ }^{1,2}$, Diana Dziewa-Dawidczyk², Oksana Wojas ${ }^{1}$, Katarzyna Retlewska ${ }^{1}$, \\ Bolesław K. Samoliński ${ }^{1}$
}

${ }^{1}$ Unit of Environmental Hazard Prevention and Allergology, Medical University of Warsaw, Warsaw, Poland

${ }^{2}$ Chair of Applied Mathematics, Faculty of Applied Informatics and Mathematics, Warsaw University of Life Sciences, Warsaw, Poland

Adv Dermatol Allergol 2020; XXXVII (6): 890-897

DOI: https://doi.org/10.5114/ada.2019.84719

\begin{abstract}
Introduction: The current state of knowledge is that allergic rhinitis can occur in two forms. One is allergic rhinitis as a manifestation of a systemic allergy with systemic atopy and positive results of skin prick tests or slgE tests. The other is local allergic rhinitis (LAR) as a local allergic reaction affecting only the nasal mucosa without systemic atopy.

Aim: To attempt to assess the usefulness of the nasal allergen provocation test for the purposes of differential diagnosis and the qualification of LAR patients for therapy.

Material and methods: The subjects in the study were a group of 6 adults diagnosed with LAR on the basis of their medical history and the results of nasal allergen provocation tests, with the allergens being house dust mites (Dermatophagoides pteronyssinus, Dermatophagoides farinae). The methods adopted in the study were a pointbased rating scale as a measure of nasal/extranasal complaints and active anterior rhinomanometry.

Results: Significant differences $(p<0.05)$ were observed, using the subjective rating scale, in relation to registered nasal and extranasal complaints in the early phase of the allergic reaction. Similarly, the rhinomanometry method revealed significant differences in nasal resistance values before and after the administration of an allergen. Conclusions: The nasal allergen provocation test is the only testing tool that objectively measures the degree of the patient's allergic reactions and is useful in qualifying LAR patients for further therapy.
\end{abstract}

Key words: local allergic reaction, nasal allergen provocation test.

\section{Introduction}

The nasal allergen provocation test (NAPT) is defined as "a method of reconstructing the response of the upper airways to natural exposure to allergens or irritants, and a method of research into the pathophysiology of diseases affecting the upper airways by testing potential biochemical mediators" [1] and plays a major role in the differential diagnosis of rhinitis conditions [2]. The specificity and sensitivity of the NAPT are high: $83.7 \%$ and $100 \%$, respectively [3]. The NAPT, as a method of measuring the degree of the allergic reaction of rhinitis patients, returns a diagnosis of allergic rhinitis (40.2\%), non-allergic rhinitis (38.8\%) and confirms local allergic reaction (LAR) [4]. In the group of patients with idiopathic rhinitis, a positive response in NAPT is manifested by $8.1-69.4 \%$ of the subjects $[5,6]$.
An extremely interesting and relatively new aspect is a local response (IgE-dependent, but without features of atopy) to an allergen in the region of the mucous membrane of the nasal cavity as well as its clinical picture obtained in the NAPT [7-10]. Local allergic reaction is regarded, in individual cases, as an early manifestation of allergic rhinitis or a separate disease in rhinitis patients. It is estimated that isolated LAR in rhinitis patients accounts for nearly $25.7 \%$ of rhinitis cases [6]. Nearly $47 \%$ of the LAR subjects are allergic to birch [11] and 62\% are allergic to the European house dust mite (Dermatophagoides pteronyssinus) [12].

\section{Aim}

The aim of this paper is to attempt to assess the usefulness of the nasal allergen provocation test for the

Address for correspondence: Oksana Wojas MD, Unit of Environmental Hazard Prevention and Allergology, Medical University of Warsaw, 1 a Banacha St, 02-097 Warsaw, Poland, phone: +48 2259910 40, e-mail: owojas@op.pl Received: 15.03.2019, accepted: 5.04.2019. 
purposes of differential diagnosis and the qualification of LAR patients for therapy. The potential risk of patients developing multimorbidity (bronchial asthma, allergic conjunctivitis) $[13,14]$ or their quality of life deteriorating and, importantly, patients developing atopy, confirms the need for research into these problems, which is increasingly often emphasised in the literature.

\section{Material and methods}

The subjects in the study were a group of 6 adults (3 females and 3 males, with the average age of 31.8 years) diagnosed with LAR on the basis of their medical history and the results of nasal allergen provocation tests, with the allergens being house dust mites (Dermatophagoides pteronyssinus, Dermatophagoides farinae). They were diagnosed at the Unit of Clinical Immunology and Allergology, University Medical Centre, Medical University of Warsaw. Patients were included in the study if their medical history confirmed allergy to house dust mites and if they have had a CT of the paranasal/frontal sinuses that ruled out inflammation, with nasal patency in the bony part of the nose. The following factors excluded patients from the study: a history of general anaphylactic reactions; contraindications to the administration of adrenaline; exacerbation of an allergic disease; severe uncontrolled bronchial asthma and/or other chronic obstructive pulmonary diseases; acute bacterial or virus infection of the nose and/or the nasal sinuses; severe forms of systemic diseases (malignant tumour, autoimmune diseases), systemic immunotherapy; ischaemic heart disease; pregnancy; age under 3 years; nasal deformities; choanal atresia; nasal septal perforation; severe nasal septum deviation; nasal polyps; atrophic rhinitis; fewer than 8 weeks after nasal surgery (particularly correction of the inferior nasal concha); acute infection of the upper airways within 2-4 weeks before the test; inflammation of the nasal/frontal sinuses $[1,2,15]$.

If the medical history taken from a patient revealed that the patient had been having nasal complaints, mainly severe, persistent nasal obstruction (Allergic Rhinitis and its Impact on Asthma - ARIA) [16], the patient's quality of life deteriorated. Furthermore, the family medical history taken from the patients confirmed a predisposition to atopy, including allergic rhinitis.

The differential diagnosis tests based on skin prick tests (for birch, grasses/corn, Dermatophagoides pteronyssinus and Dermatophagoides farinae, moulds 1 (Botrytis cinerea, Cladosporium herbarium, Alternaria tenuis, Curvularia lunata, Fusarium moniliforme, Helminthosporium), moulds 2 (Aspergillus fumigates, Mucor mucedo, Penicillium notatum, Pullularia pullulans, Rhizopus nigricans, Serpula lacrymans), dog, cat, Cladosporium herbarium and Alternaria tenuis moulds) and slgE tests, ruled out atopy. No features of bronchial tree obstruction were revealed in a spirometry test (Lungtest 1000, MES).
The method used in the study was the NAPT with a standard dose of the Allergopharma allergen (5000 $\mathrm{SBU} / \mathrm{ml})$. The allergen $(0.2 \mathrm{ml})$ was inhaled into both nostrils at room temperature, by means of an atomizer. The patients' nasal complaints were measured on a point-based rating scale (for nasal and extranasal symptoms). Rhinomanometry (Rhinotest, MES) was also used, in accordance with the test protocol (see appendix) (flow rate: $\pm 18 \mathrm{l} / \mathrm{s}$, accuracy of flow rate measurement: < $2 \%$, volume measurement resolution: $\pm 10 \mathrm{ml} / \mathrm{s}$, measured pressure range: $\pm 1.25 \mathrm{kPa}$, accuracy of pressure measurement: $\pm 1 \%$, pressure measurement resolution: $\pm 1 \mathrm{~Pa}$, measuring head: MES/type DV 40, dead area: DV $40-40 \mathrm{ml}$, head resistance: DV $40<0.2 \mathrm{~cm} \mathrm{H}_{2} \mathrm{O} / \mathrm{l} / \mathrm{s}$ (with a flow rate of $1 \mathrm{l} / \mathrm{s}$ )). The rhinomanometry test included an analysis of nasal resistance (using the standard and Brom's methods) examination (Rn RSIn kPa/l/s = standard right-side nasal resistance during inspiration; $\mathrm{Rn} \mathrm{RBIn} \mathrm{kPa} / \mathrm{l} / \mathrm{s}=$ Brom's right-side nasal resistance during inspiration, Rn RSEx $\mathrm{kPa} / \mathrm{l} / \mathrm{s}=$ standard right-side nasal resistance during expiration; Rn RBEx $\mathrm{kPa} / \mathrm{l} / \mathrm{s}=$ Brom's right-side nasal resistance during expiration; $\mathrm{Rn}$ $\mathrm{LSIn} \mathrm{kPa} / \mathrm{l} / \mathrm{s}=$ standard left-side nasal resistance during inspiration; Rn LBIn kPa/l/s = Brom's left-side nasal resistance during inspiration; Rn LSEx kPa/l/s = standard leftside nasal resistance during expiration; Rn LBEx kPa/l/s = Brom's left-side nasal resistance during expiration) for the measured flow rate values (V, SD), separately for the right and left sides during normal breathing.

The test was carried out three times: after the patient's adaptation to the conditions in the laboratory (20 min, ambient temperature: $21^{\circ} \mathrm{C}$, relative humidity: 40-50\%), which was the initial, or baseline measurement; after the administration of control solution ( $0.9 \%$ $\mathrm{NaCl}+0.4 \%$ Pheno - the carrier for the test substance); and after local administration of the allergen. The early phase of the allergic reaction was evaluated 20 min after the local administration of the allergen [1, 2]. Patients with nasal symptoms measured as 5 points on the pointbased rating scale, with an increase by $40 \%$ of the air flow resistance rate before the administration of the allergen, were excluded from participation in the study. The NAPT was performed on an outpatient basis by qualified medical personnel, in accordance with all the applicable safety standards [15].

\section{Statistical analysis}

The statistical analysis for the measured values of nasal resistance ( $R n R S I n, R n R B I n, R n R S E x, R n R S E x, R n L S I n$, $R n L B / n, R n L S E x, R n L B E x)$ and for the point-based rating scale at each stage of the test was performed using the Shapiro-Wilk test of normality, the Wilcoxon signed-rank test and analysis of variance (ANOVA), with a significance level of $\alpha=0.05$. Also, box plots were used to depict differences in nasal resistance values between the different stages of the test. Additionally, ROC curves were used 
to assess the specificity and sensitivity of the tools for measuring the response of the nasal mucosa to the allergens administered.

\section{Results}

Highly positive values in the NAPT, based on European Academy of Allergy and Clinical Immunology (EAACI) guidelines [15], were recorded for 5 subjects (one subject demonstrated a moderately positive value; nasal resistance: $25 \%$, 3 points on the point-based rating scale). Significant differences were recorded, using the point-based rating scale, in relation to registered nasal and extranasal complaints in the early phase of the allergic reaction (Figure 1). Two of the six subjects demonstrated nasal obstruction as early as at the second stage of the test. The swelling of the nasal mucosa increased significantly after the administration of the allergen. As regards extranasal symptoms, two of the six subjects demonstrated coughing in the early phase of the allergic reaction.

Similarly, the rhinomanometry method revealed significant differences in nasal resistance values before and after the administration of an allergen. The average values and the standard deviation values for the current value, the variability coefficient $(\mathrm{V})$ and the deviation (S) values for the different nasal resistance values are presented in Table 1. In 2 cases, due to technical problems, i.e. considerable nasal obstruction, it was possible to carry out the test only at 5-minute intervals. The Wilcoxon signed-rank test revealed a difference between the average current values before and after the NAPT; for $R n L S I n$, in the first test versus the value after the local administration of the allergen $(p=0.025970)$ and in the second test in relation to the values recorded in the early phase of the allergic reaction ( $p=0.04113)$. Similarly, an analysis of the $R n L B / n$ nasal resistance values revealed differences between the values measured in the initial (baseline) test versus those measured after the NAPT ( $p=0.02597)$ (Table 1).

Additionally, an analysis of a ROC curve (including the AUC field) for the current value was performed (the patients were divided into two groups: measurements in the first and second tests (no complaints measured) and measurements when complaints occurred (nasal obstruction after the NAPT). Differences between the patients included in the study were the most evident in the case of the following values: $R n L S \ln (A \cup C=0.875)$, $R n L B I n(A \cup C=0.819), \operatorname{RnLBEX}(A \cup C=0.792), R n L S E x$ $(A \cup C=0.75)$ (Figure 2).

\section{Discussion}

The NAPT, with measurements in the early and late phases of the allergic reaction made using standardised techniques including acoustic rhinometry, rhinomanometry, peak nasal inspiratory flow (PNIF), the nasal symptom score scale (VAS) [15] and other techniques: the content of reaction mediators in nasal lavage fluid (slgE, tryptase, and eosinophilic cationic protein (ECP) or the level of nitrogen oxide in the air exhaled), plays a major role in the process of qualifying patients for further therapy. The results of NAPT can sometimes be difficult to evaluate and interpret, when the wrong nasal patency measurement technique was used. The most specific and sensitive techniques include: acoustic rhinometry, optical rhinomanometry and PNIF. In our report, we have proved, on the one hand, the specific usefulness and relatively good sensitivity and specificity of the technique
$\mathbf{A}_{14}$

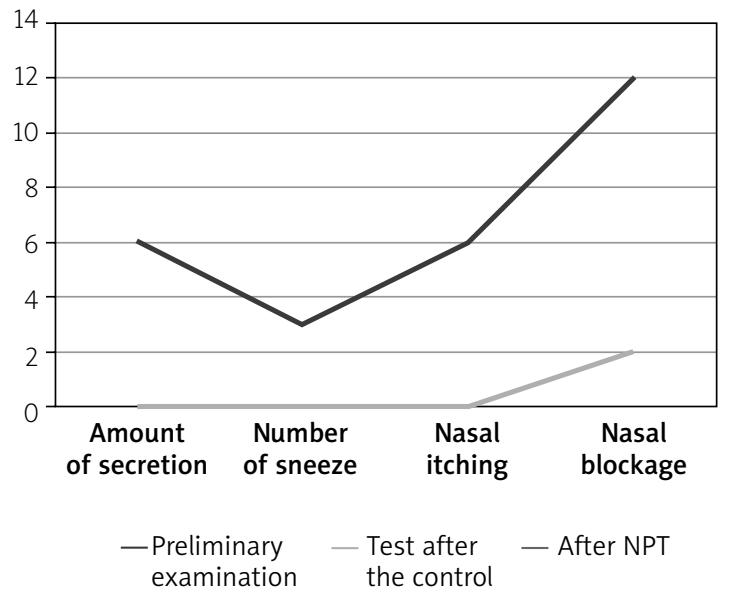

B 4.5

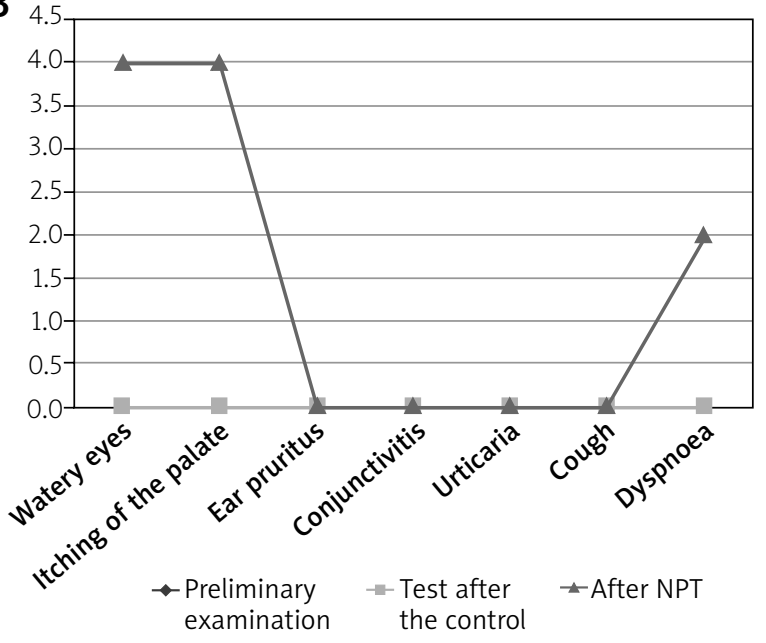

Figure 1. Subjective assessment of nasal and extranodal ailments in the early phase of an allergic reaction of a nasal allergen provocation test: $\mathbf{A}$ - Nasal symptoms of the early phase of the allergic reaction, $\mathbf{B}$ - the extra-nasal symptoms of the early allergic reaction phase 
employed in our study, namely rhinomanometry, and, on the other, the fact that it is technically difficult to obtain the following current values: nasal resistance in connection with nasal obstruction (in the case of highly positive results of the NAPT, nasal obstruction means failure to reach the required resistance value for the pressure values of 150 or $100 \mathrm{kPA}$, while watery discharge from the nose prevents patches from being stuck onto the nostrils) $[16,17]$.

The NAPT, which imitates the natural response observed under natural conditions, objectivises a number of other accompanying variables, including visual symptoms (subjective assessment using, for example, the VAS scale), plus a spirometry test before and after the administration of an allergen, is a source of valuable information on the condition of the bronchial tree and a precise measure of the response to the presence of an allergen in the respiratory system. This is particularly important given the fact that allergic rhinitis co-exists with bronchial asthma in nearly $40 \%$ of patients [18]. Nearly $30 \%$ of LAR patients experience problems regarding the lower airways, which may indicate co-existence of bronchial asthma [6, 19, 20]. In a bronchial provocation test with the Dermatophagoides pteronyssinus allergen, 8 of 16 LAR subjects demonstrated an increase in the content of eosinophils and ECP in bronchial lavage fluid, which confirms the hypothesis that LAR co-exists with bronchial asthma [21]. Similarly, a conjunctival provocation test in the case of LAR patients determines a typical picture of sensitivity to the allergen administered and confirms the presence of ECP in the fluid discharged from the eye [22].

In differential diagnosis, NAPT is used to decide whether or not to qualify subjects for therapy in the case of clinical and diagnostic differences in the qualification process, e.g. for specific immunotherapy (with an allergic disease confirmed by standard tests) and should be regarded as the gold standard. However, if skin prick tests or slgE tests have confirmed no features of atopy, the NAPT is not used (Figure 3). Moreover, if the results of tests are negative, as is the case in LAR, the patient is often diagnosed with non-allergic rhinitis. Rhinitis is an inflammation of the nasal mucosa. Symptoms of rhinitis include water discharge from the nose, sneezing or a feeling of nasal obstruction. These symptoms must continue for more than an hour a day and for many days a year. Rhinitis can be caused by various factors: allergic or non-allergic, infection-related, hormonal, pharmacological, occupational, as well as by irritants. Allergic rhinitis is an IgE-dependent inflammation of the nasal mucosa. It is caused by exposure to an allergen and

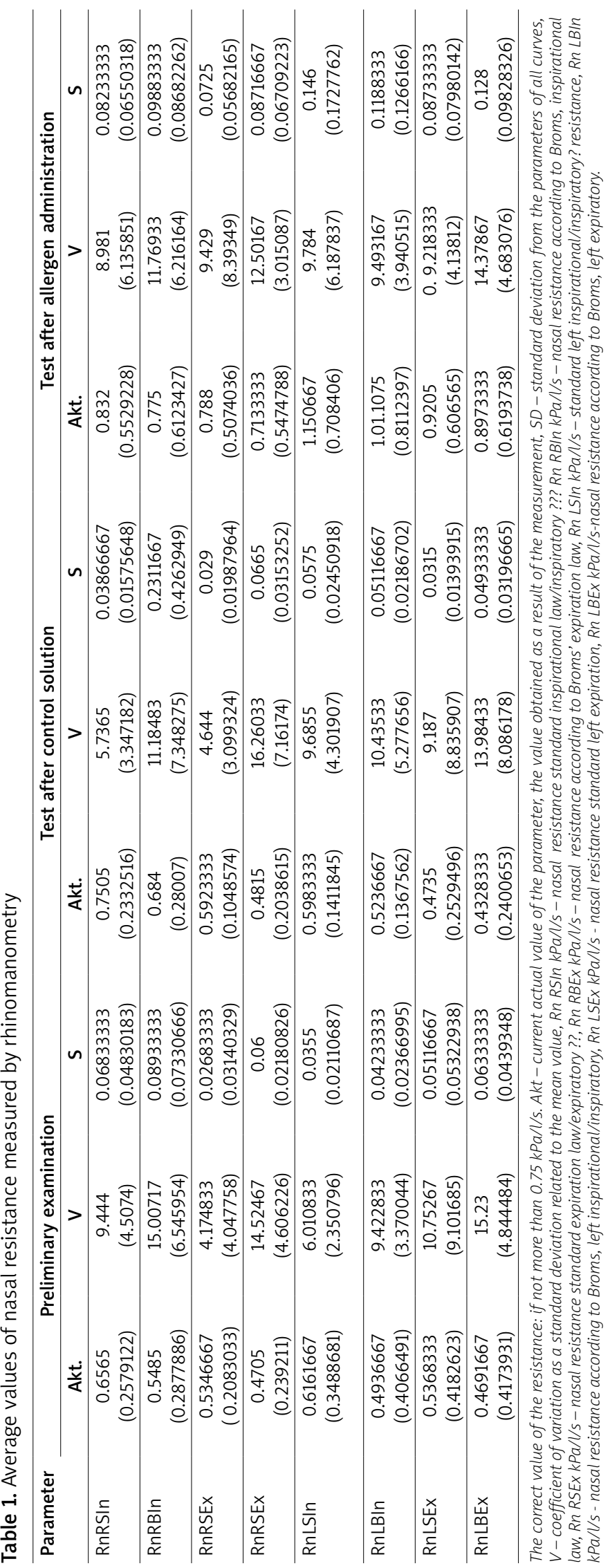



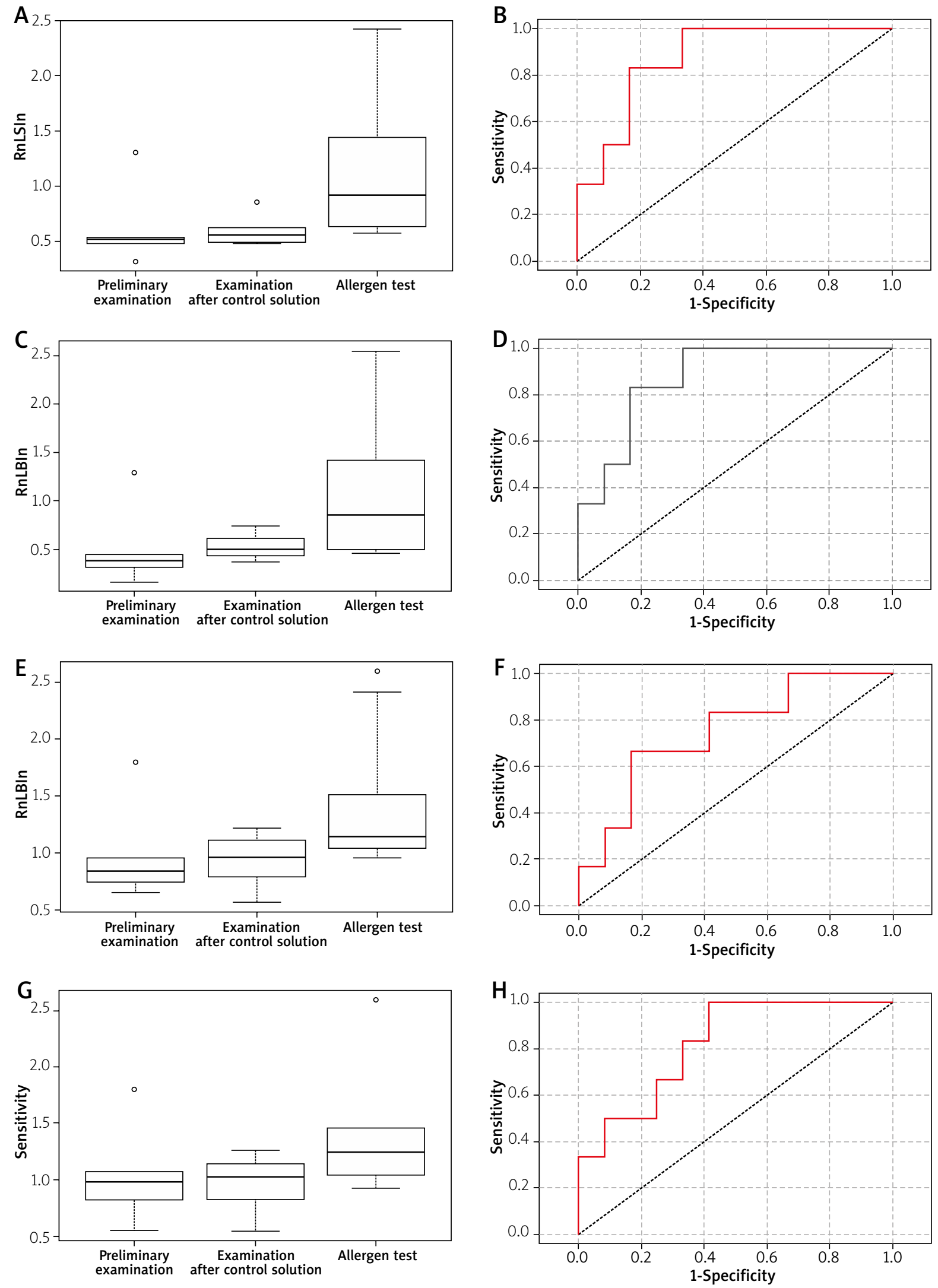

Figure 2. Selected values of the rhinomanometer curve in the early allergic reaction phase: A - Boxplot for values $R n L S / n$, B - the ROC curve for the parameter $R n L S \ln (A \cup C=0.875), C$ - boxplot for values $R n L B / n, D$ - the ROC curve for the parameter RnLBIn (AUC =0.819), $\mathrm{E}$ - boxplot for values RnLBEX, $\mathrm{F}$ - the ROC curve for the parameter RnLBEx $(A U C=0.792)$, $\mathrm{G}$ - boxplot for values RnLSEX, $\mathrm{H}$ - the ROC curve for the parameter RnLSEX (AUC $=0.75$ ) 


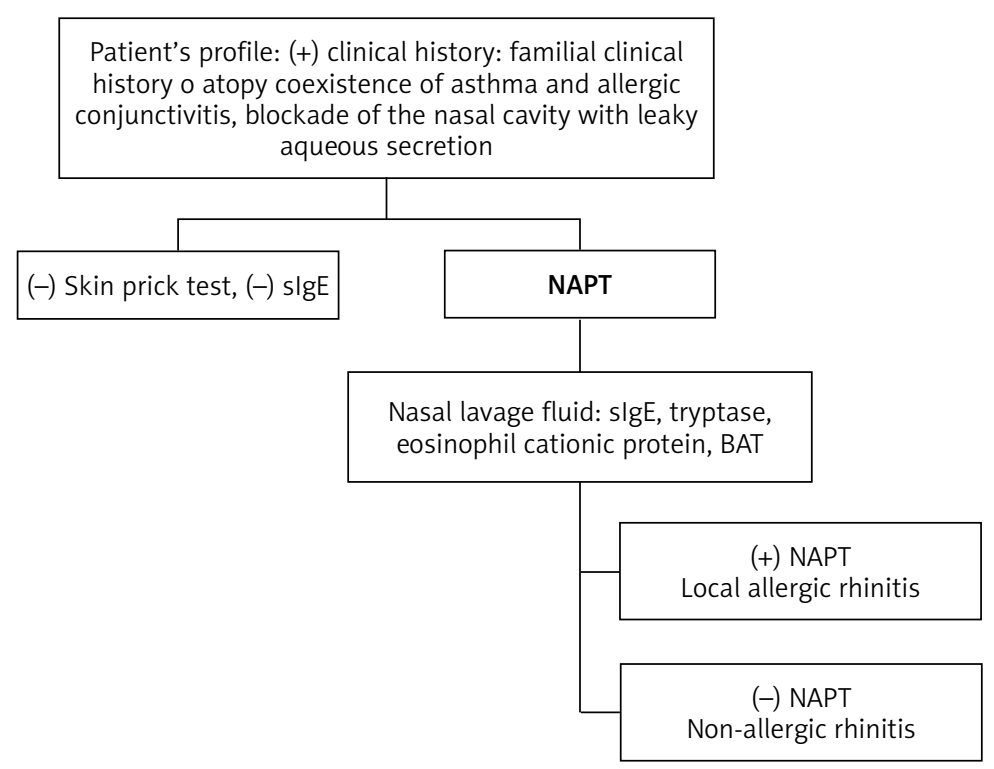

Figure 3. Nasal allergen provocation test in the diagnosis of local allergic rhinitis (adopted from Campo P, Rondon C, et al. $[21,25])$

is the most common form of non-infectious rhinitis [16, $23,24]$. ARIA classifies allergic rhinitis by the duration of symptoms into: periodic allergic rhinitis (symptoms occurring for fewer than 4 days a week or for fewer than 4 weeks), which is normally caused by plant pollen allergens and/or mould spore allergens, and chronic allergic rhinitis (symptoms occurring for more than 4 days a week and/or more than 4 weeks). The latter is usually caused by allergens permanently present in the patient's environment (house dust mites, moulds, animal allergens). By the severity of symptoms, allergic rhinitis is classified into mild rhinitis (mild symptoms which do not significantly affect the patient's quality of life and/ or daily activities; medical advice is not usually sought); moderate rhinitis (symptoms affecting the patient's daily activities (work, school, physical activity) and causing sleep problems); the patient is usually determined to seek medical advice); severe rhinitis (symptoms preventing the patient from going about their daily activities and causing serious sleep problems; the patient's quality of life is clearly worse and the patient is determined to seek medical advice) [16].

The current state of knowledge is that allergic rhinitis can occur in two forms. One is allergic rhinitis as a manifestation of a systemic allergy with systemic atopy and positive results of skin prick tests or slgE tests. The other is a LAR as a local allergic reaction affecting only the nasal mucosa without systemic atopy (known as 'entopy'). Local allergic rhinitis is a specific phenotype of rhinitis. Its symptoms are similar to those of allergic rhinitis, with a local inflammatory response mediated by $\mathrm{Th}_{2}$ lymphocytes, the production of allergen-specific IgE antibodies, and with a positive response in NAPT and no slgE in serum and on mast cells [20].

Research into LAR began in the 1970s. In 1975, Huggings and Brostoff proved the presence of slgE in the nasal lavage fluid in the case of patients with symptoms of allergic rhinitis and negative results of allergy tests [25]. A year later, Johansson and Deuschl described a laboratory method of detecting the presence of slgE in the nasal lavage fluid in the case of patients with negative results of allergy tests [26]. In 2003, Powe et al. proposed, in contrast to atopy, a new term, "entopy", which refers to local production of slgE in the mucous membrane of the respiratory tract [27]. The definition of LAR was first proposed by Carmen Rondon, an outstanding researcher into this condition. Drawing on many years of research into local allergic rhinitis, Rondon et al. produced a clinical profile of a LAR patient, i.e. usually a young female non-smoker living in a large city, with year-round symptoms of severe rhinitis (with negative results of allergy tests; skin prick tests and slgE). Her symptoms include, in particular, nasal obstructions, sneezing, itching and abundant nasal discharge. In $36 \%$ of patients, the first symptoms occurred in the early childhood. The patient's family medical history usually confirms a predisposition to atopy. Local allergic rhinitis is very often accompanied by allergic conjunctivitis and bronchial asthma. The patient profile based on our review of the literature corresponds with the profile based on our study. What is important is that all the subjects demonstrated significant changes in nasal patency as measured using rhinomanometry, while the accompanying symptoms in the upper and lower airways and the eyes emphasise the need to use NAPT in differential diagnosis for $\operatorname{LAR}[6,11,19,20]$. 
It is not certain whether LAR is a separate condition or merely an "introduction" to the development of the classic allergic rhinitis. Research by Gelardi et al. suggests that local production of IgE may be a part of a nonspecific immune response to environmental factors, and the researchers recommend that great care should be taken in the diagnosis of LAR [28].

In the case of patients with symptoms of allergic rhinitis and negative results of skin prick tests and with slgE in serum, the diagnosis of LAR is based on a positive result of the nasal allergen provocation test. NAPT normally involves the use of one allergen. However, Rondon et al. proposed a NAPT protocol that involves administering a few allergens at short intervals. They claim that this test method is safe, specific, sensitive and takes less time. Other diagnostic tests for LAR include measurement of the content of slgE, tryptase and ECP in the nasal discharge fluid, and basophil activation tests (BATs) $[11,19,20]$. It is important to note that the specificity of different NAPT evaluation tools varies greatly, e.g. for the nasal discharge fluid evaluation, specificity is $22 \%$ and sensitivity is $40 \%[25,29,30]$.

On the therapy side, LAR is treated with pharmaceuticals: second-generation antihistamines and nasal glucocorticosteroids. Specific immunotherapy is considered as an option in the treatment of LAR more and more frequently. However, the latest guidelines do not recommend this method as scientific research in support of its use is not sufficient. Based on a randomised double blind placebo-controlled study, Rondon et al. presented encouraging results regarding the use of specific immunotherapy in the treatment of LAR in patients with allergies to grass and house dust mites [31, 32]. Similarly, other studies indicate that allergens are better tolerated in NAPT after a course of immunotherapy as compared to before such therapy, and for nearly $50 \%$ of the subjects, the results of NAPT were negative [32]. It seems that the NAPT is the most important test for qualifying patients for specific immunotherapy. It is important to stress that current guidelines do not refer to LAR as an indication for specific immunotherapy, and further research is needed before this method can be used.

\section{Conclusions}

The NAPTs performed on an outpatient basis or with the use of mobile allergen challenge chambers provide valuable information on the severity of the subjects' allergies to common environmental allergens, including for patients with LAR. On the one hand, NAPTs determine whether a subject should be included or excluded from further therapy. On the other, it is the only objective method for individualising an allergic disease if clinical differences occur.

\section{Conflict of interest}

The authors declare no conflict of interest.

\section{References}

1. Samoliński B, Rapiejko P, Krzych-Fałta E, et al. Standardy wykonywania donosowych prób prowokacyjnych. Adv Dermatol Allergol 2010; 27: 141-60.

2. Samoliński B, Krzych-Fałta E. Donosowa próba prowokacyjna z alergenem. Alergol Pol 2018; 5: 192-6.

3. Mazurek H, Modrzynski M, Krzych-Falta E. Nasal challenge tests with allergens versus other allergy and rhinitis diagnostic tests. Adv Dermatol Allergol 2010; 27: 193-5.

4. Bożek A, Ignasiak B, Kasperska-Zając A, et al. Local allergic rhinitis in elderly patients. Ann Allergy Asthma Immunol 2015; 114: 199-202.

5. Cheng KJ, XU YY, Liu HY, et al. Serum eosinophil cationic protein level in Chinese subjects with nonallergic and local allergic rhinitis and its relation to the severity of disease. Am J Rhinol Allergy 2013; 27: 8-12.

6. Rondon C, Campo P, Galindo L, et al. Prevalence and clinical relevance of local allergic rhinitis. Allergy 2012; 67: 1282-8.

7. Campo P, Salas M, Blanca-Lopez N, et al. Local allergic rhinitis. Immunol Allergy Clin N Am 2016; 36: 321-32.

8. Bozek A, Scierski W, Ignasiak B, et al. The prevalence and characteristics of local allergic rhinitis in Poland. Rhinology 2018 Dec 17. doi: 10.4193/Rhin18.137.

9. Hamizan AW, Rimmer J, Husain S, et al. Local specific immunoglobulin E among patients with nonallergic rhinitis: a systematic review. Rhinology 2019; 57: 10-20.

10. Rondón C, Eguiluz-Gracia I, Campo P. Is the evidence of local allergic rhinitis growing? Curr Opin Allergy Clin Immunol 2018; 18: 342-9.

11. Wedback A, Enbom H, Eriksson NE, et al. Seasonal ono-allergic rhinitis (SNAR) - a new disease entity? A clinical and immunological comparison between SNAR, seasonal allergic rhinitis and persistent non-allergic rhinitis. Rhinology 2005; 45: 86-92.

12. Carney AS, Powe DG, Huskisson RS, et al. Atypical nasal challenge in patients with itiopathic rhinitis: more evidence for the existence of allergy in the absence of atopy? Clin Exp Allergy 2002; 32: 1436-40.

13. Raciborski F, Bousqet J, Namysłowski A, et al. Dissociating polysensitization and multimorbidity in children and adults from a Polish general population cohort. Clin Transl Allergy 2019; 9: 4.

14. Augé J, Vent J, Agache I, et al. EAACI Position paper on the standardization of nasal allergen challenges. Allergy 2018; 73: 1597-608.

15. Bousquet J, Khaltaev N, Cruz A, et al. Allergic Rhinitis and its Impact on Asthma (ARIA) 2008 update (in collaboration with the World Health Organization, GA(2)LEN and AllerGen). Allergy 2008; 63 Suppl 86: 8-160.

16. Krzych-Fałta E, Samliński B. Objectification of the nasal patency assessment techniques used in nasal allergen provocation testing. Adv Dermatol Allergol DOI: https://doi. org/10.5114/ada.2019.81404.

17. Samoliński B, Sybilski A. Znaczenie stanów zapalnych błony śluzowej nosa w astmie oskrzelowej. Adv Dermatol Allergol 2010; 27: 223-9.

18. Rondon C, Campo P, Zambonino MA, et al. Follow-up study in local allergic rhinitis shows a consistent entity not evolving to systematic allergic rhinitis. J Allergy Clin Immunol 2014; 133: 1026-31.

19. Rondon C, Campo P, Togias A, et al. Local allergic rhinitis: mechanism, diagnosis and prevalence for occupational rhinitis. Curr Opin Allergy Clin Immunol 2015; 15: 111-6. 
20.Campo P, Antunez C, Rondon C, et al. Positive bronchial challenges to $\mathrm{D}$. pteronyssinus in asthmatic subjects in absence of systematic atopy. J Allergy Clin Immunol 2011; 127 (suppl 2): AB6.

21. Leonardi A, Vattista MC, Gismondi M, et al. Antigen sensitivity evaluated by tearspecific and serum-specyfic IgE, skin test, and conjunctival and nasal provocation tests in patients with ocular allergic diseases. Eye 1993; 7: 461-4.

22. Pillai P, Fang C, Chan YC, et al. Mite allergen-specific lgE is detectable in the bronchial mucosa of nonatopic asthmatic patients. J Allergy Clin Immunol 2014; 133: 1770-2.e.1.

23. Samoliński B, Arcimowicz M, Buczyłko K, et al. Polskie Standardy Leczenia Nieżytów Nosa (PoSLeNN). Stanowisko Panelu Ekspertów Polskiego Towarzystwa Alergologicznego. Medycyna Praktyczna, Krakow 2013.

24. Campo P, Rondon C, Gould HJ, et al. Local lgE in non-allergic rhinitis. Clin Exp Allergy 2015; 45: 872-81.

25. Huggins KG, Brostoff J. Local production of specific IgE antibodies in allergic-rhinitis patients with negative skin tests. Lancet 1975; 2: 148-50.

26. Johansson SG, Deuschl H. Immunoglobulins in nasal secretion with special reference to IgE. Methodological studies. Int Arch Allergy Appl Immunol 1976; 52: 364-75.

27. Powe DG, Jagger C, Kleinjan A et al. "Entopy": localized mucosal allergic disease in the absence of systemic responses for atopy. Clin Exp Allergy 2003; 33: 1374-9.

28. Gelardi M, Guglielmi AVN, lannuzzi L, et al. Local allergic rhinitis: entopy or spontaneous response? World Allergy Organ J 2016; 9: 39.

29. Rondon C, Romero JJ, Lopez S, et al. Local IgE production and positive nasal provocation test in patients with persistent nonallergic rhinitis. J Allergy Clin Immunol 2007; 119: 899905.

30. Rondon C, Dona I, Lopez S, et al. Seasonal idiopathic rhinitis with local inflammatory response and specific lgE in absence of systemic response. Allergy 2008; 63: 1352-8.

31. Rondon C, Blanca-Lopez N, Campo P, et al. Specific immunotherapy in local allergic rhinitis: a randomized, double-blind placebo-controlled trial with Phleum pretense subcutaneous allergen immunotherapy. Allergy 2018; 73: 905-15.

32. Rondon C, Campo P, Salas M, et al. Efficacy and safety of D. pteronyssinus immunotherapy in local allergic rhinitis: a double-blind placebo-controlled clinical trial. Allergy 2016; 71: 1057-61. 